\title{
Crystal Structure and Comparative Sequence Analysis of GmhA from Colwellia psychrerythraea Strain 34H Provides Insight into Functional Similarity with DiaA
}

\author{
Hackwon Do', Ji-Sook Yun ${ }^{2}$, Chang Woo Lee ${ }^{1,3}$, Young Jun Choí, Hye-Yeon Kim ${ }^{4}$, Youn-Jung Kim, \\ Hyun Park ${ }^{1,3}$, Jeong Ho Chang ${ }^{2, *}$, and Jun Hyuck Lee ${ }^{1,3, *}$
}

\begin{abstract}
The psychrophilic organism Colwellia psychrerythraea strain $34 \mathrm{H}$ produces extracellular polvsaccharide substances to tolerate cold environments. Sedoheptulose 7phosphate isomerase (GmhA) is essential for producing Dglycero-D-mannoheptose 7-phosphate, a key mediator in the lipopolysaccharide biosynthetic pathway. We determined the crystal structure of GmhA from C. psychrerythraea strain 34H (CpsGmhA, UniProtKB code: Q47VU0) at a resolution of $2.8 \AA$. The tetrameric structure is similar to that of homologous GmhA structures. Interestingly, one of the catalytic residues, glutamate, which has been reported to be critical for the activity of other homologous GmhA enzymes, is replaced by a glutamine residue in the CpsGmhA protein. We also found differences in the conformations of several other catalytic residues. Extensive structural and sequence analyses reveal that CpsGmhA shows high similarity to Escherichia coli DnaA initiatorassociating protein $A$ (DiaA). Therefore, the CpsGmhA structure reported here may provide insight into the structural and functional correlations between GmhA and DiaA among specific microorganisms.
\end{abstract}

\section{INTRODUCTION}

Psychrophilic organisms are cold-adapted microorganisms that have overcome the constraints associated with living in permanently cold environments (Pikuta et al., 2007; Russell, 1990).

${ }^{1}$ Division of Polar Life Sciences, Korea Polar Research Institute, Incheon 406-840, Korea, ${ }^{2}$ Department of Biology Education, Kyungpook National University, Daegu 702-701, Korea, ${ }^{3}$ Department of Polar Sciences, University of Science and Technology, Incheon 406-840, Korea, ${ }^{4}$ Protein Structure Group, Korea Basic Science Institute, Chungbuk 363-883, Korea, ${ }^{5}$ Department of Marine Science, Incheon National University, Incheon 406-772, Korea

*Correspondence: junhyucklee@kopri.re.kr (JHL); jhcbio@knu.ac.kr (JHC)

Received 2 July, 2015; revised 1 September, 2015; accepted 15 September, 2015; published online 26 November, 2015

Keywords: Colwellia psychrerythraea strain 34H, CpsGmhA, DiaA, psychrophile, sedoheptulose 7-phosphate isomerase
Cold environments can induce molecular changes in an organism, including abnormalities such as low enzymatic activity, protein denaturation, and mis-folding of proteins (Russell, 1998). In order to overcome these deficiencies, several coldadaptation mechanisms have been described, including coldactive enzymes, an unsaturated fatty-acid membrane, antifreeze proteins, and sugar and/or alcohol compounds such as glycerol, trehalose, and sorbitol (Chattopadhyay, 2006; Feller, 2013; Feller and Gerday, 2003).

One of the most interesting cold-adaptation mechanisms is biofilm formation using extracellular polysaccharide substances (EPSs) that are found in the O-antigen component of lipopolysaccharides (LPS) and cell-bound polysaccharides (Bazaka et al., 2011). The EPSs play important temperature-buffering and cryoprotectant roles in cold-adapted microorganisms (Bazaka et al., 2011; Krembs et al., 2002). The psychrophilic bacterium Colwellia psychrerythraea strain $34 \mathrm{H}$ is a well-known producer of EPSs, which function as cryoprotectants (Marx et al., 2009). Increased production of EPSs is observed when samples are frozen from -8 to $-14^{\circ} \mathrm{C}$, which represents a strategy similar to that used by yeasts that increase the production of cryoprotectants (polyols and/or sugar alcohol) in response to osmotic stress (Marx et al., 2009; Shen et al., 1999). Recently, the molecular structure of capsular polysaccharides synthesized from C. psychrerythraea strain $34 \mathrm{H}$ was determined and characterized. Interestingly, it resembles other known antifreeze (Glyco) proteins and appears to inhibit ice recrystallization (Carillo et al., 2015).

C. psychrerythraea strain $34 \mathrm{H}$ was initially isolated from Arctic marine sediments (Huston et al., 2000). The temperature of growth ranges from $-12^{\circ} \mathrm{C}$ to $19^{\circ} \mathrm{C}$ and its optimal growth temperature is $8^{\circ} \mathrm{C}$ to $9^{\circ} \mathrm{C}$ (Wells and Deming, 2006). The results of genomic sequence analyses show that many gene products of $C$. psychrerythraea strain $34 \mathrm{H}$ play a role in the production and secretion of EPSs (Methe et al., 2005). These EPSs stabilize an extracellular protease, ColAP, against thermal denaturation (Huston et al., 2004). In addition, EPSs are involved in regulating intracellular biosynthetic reactions to proceed at extremely low temperatures; however, the external conditions that activate the production of EPSs remain unknown (Junge et al., 2006).

Gram-negative bacteria, including C. psychrerythraea strain 
$34 \mathrm{H}$, contain either LPS or capsular polysaccharides (CPSs) in their outer membrane. Typical LPS is comprised of three covalently linked domains: lipid $A$, core oligosaccharide, and, in some bacteria, O-antigen. The core oligosaccharide is composed of inner and outer domains; the inner core contains 3deoxy-D-manno-oct-2-ulosonic acid (Kdo), which is bound to lipid A, and L-glycero-D-manno heptose (heptose), which is linked to 3-deoxy-D-manno-oct-2-ulosonic acid (Brooke and Valvano, 1996; Nikaido, 2003; Raetz and Whitfield, 2002). Heptose is the connecting point for the variable outer-core saccharides that serve as the binding sites for O-antigen to produce complete LPS. Sedoheptulose 7-phosphate isomerase (GmhA), encoded by $g m h A$, catalyzes the conversion of sedoheptulose 7-phosphate (S7P) to D-glycero-D-mannoheptose 7-phosphate (M7P) in the first step of the heptose biosynthesis pathway (Kneidinger et al., 2001). Aberrant GmhA in Escherichia coli does not synthesize heptose and produces truncated LPS owing to the lack of a connecting point for more distal moieties (Brooke and Valvano, 1996).

The structure and catalytic mechanisms of GmhA from various species have been characterized because $\mathrm{GmhA}$ is considered an important target for antibiotic development (Harmer, 2010; Kim and Shin, 2009; Seetharaman et al., 2006; Taylor et al., 2008). In this study, we determined the crystal structure of GmhA from C. psychrerythraea strain 34H (CpsGmhA), which is critical for LPS biosynthesis and activity, and is potentially maintained at low temperatures for production of polysaccharides. In addition, sequence analysis reveals that CpsGmhA has high similarity with $E$. coli DnaA initiator-associating protein A (DiaA). Therefore, characterization of the structure of
CpsGmhA may provide insight into the structural and functional correlations between GmhA and DiaA among microorganisms; additionally, comparisons of these proteins in mesophilic and psychrophilic organisms may help to elucidate the mechanism of low temperature-adapted enzymes.

\section{MATERIALS AND METHODS}

\section{Cloning, protein expression, and purification}

The gene encoding GmhA protein (UniProtKB code: Q47VU0, amino acids 1-196) was amplified by polymerase chain reaction (PCR) from the genomic DNA of $C$. psychrerythraea strain $34 \mathrm{H}$ and subcloned into the pET $28 \mathrm{a}(+)$ vector (Novagen, USA). PCR amplification of the gene was performed using primers containing Ndel and Xhol restriction enzyme sites: forward primer 5'- CGATAACATATGCTAGAACAGATCAAAAAC-3' and reverse primer 5'- CGATAACTCGAGTCATGAATCACCTTGCG GGAA-3'. E. coli BL21(DE3) cells were transformed with the cloned $\mathrm{pET} 28 \mathrm{a}(+)$ vector, which contains an N-terminal 6histidine (His) tag and a thrombin cleavage site. The E. coli cells were grown at $37^{\circ} \mathrm{C}$ until the cultures reached an optical density of approximately 0.7 at $600 \mathrm{~nm}$. Heterologous expression of the target protein was induced using $1 \mathrm{mM}$ isopropyl- $\beta$ D-thiogalactopyranoside (Bioneer, Korea) at $25^{\circ} \mathrm{C}$ overnight, and the expressed protein was collected by centrifugation at $7000 \mathrm{rpm}$ for $20 \mathrm{~min}$. The cell pellet was resuspended in lysis buffer (50 mM sodium phosphate [pH 8.0], $300 \mathrm{mM} \mathrm{NaCl}$, and $5 \mathrm{mM}$ imidazole) containing phenylmethane sulfonyl fluoride and disrupted by sonication (Vibra-Cell VCX400; Sonics \& Materials, Inc., USA).

Table 1. X- Ray data collection and refinement statistics for CpsGmhA

\begin{tabular}{|c|c|}
\hline & CpsGmhA \\
\hline \multicolumn{2}{|l|}{ Data collection } \\
\hline X-ray source & PLS-5C \\
\hline Space group & $\mathrm{C} 222$ \\
\hline Unit-cell parameters $(\AA)$ & $a=73.3, b=109.3, c=44.3$ \\
\hline Wavelength $(\AA)$ & 0.97951 \\
\hline Resolution Range $(\AA)$ & $44.3-2.8(2.95-2.80)$ \\
\hline No. of observed reflections & $54978(8311)$ \\
\hline No. of unique reflections & $4643(671)$ \\
\hline Completeness (\%) & $99.9(100)$ \\
\hline Redundancy & $6.4(6.5)$ \\
\hline$R_{\text {merge }}{ }^{\text {a }}$ & $0.136(0.315)$ \\
\hline$I / \sigma$ & $13.0(7.3)$ \\
\hline \multicolumn{2}{|l|}{ Refinement } \\
\hline Resolution range & $44.3-2.8(3.53-2.80)$ \\
\hline Reflections: working/free & $4643 / 215$ \\
\hline$R_{c r y s t} \mathrm{~b}$ & $0.210(0.234)$ \\
\hline$R_{\text {free }}{ }^{\mathrm{C}}$ & $0.280(0.341)$ \\
\hline \multicolumn{2}{|l|}{ Ramachandran plot: } \\
\hline favored/ allowed/disallowed (\%) ${ }^{d}$ & $94.8 / 5.2 / 0$ \\
\hline R.m.s.d. bonds $(\AA)$ & 0.001 \\
\hline R.m.s.d. angles $\left({ }^{\circ}\right)$ & 0.381 \\
\hline PDB accession code & $5 \mathrm{BY} 2$ \\
\hline
\end{tabular}

${ }^{\mathrm{a}} R_{\text {merge }}=\sum|<|>-|| \mid / \Sigma<1>$.

${ }^{\mathrm{b}} R_{\text {cryst }}=\sum|| \mathrm{Fo}|-| \mathrm{Fc}|| / \sum|\mathrm{Fo}|$.

${ }^{\mathrm{c}} R_{\text {free }}$ calculated with $5 \%$ of all reflections excluded from refinement stages using high-resolution data.

${ }^{\mathrm{d}}$ The Ramachandran plot was calculated using MolProbity (http://molprobity.biochem.duke.edu/). Values in parentheses indicate the statistics for the highest resolution shells. 
Crystal Structure of Putative CpsGmhA

Hackwon Do et al.

Table 2. Sequence information of the consensus cladogram

\begin{tabular}{|c|c|c|}
\hline Protein & Species & NCBI reference sequence \\
\hline \multirow[t]{11}{*}{ GmhA } & Colwellia psychrerythraea & Q47VU0.1 \\
\hline & Escherichia coli & NP_308276.1 \\
\hline & Campylobacter jejuni & WP_002858021.1 \\
\hline & Pseudomonas aeruginosa & WP_016253868.1 \\
\hline & Burkholderia pseudomallei & WP_011205222.1 \\
\hline & Vibrio cholera & WP_000284054.1 \\
\hline & Leptospira alstonii & WP_020772754.1 \\
\hline & Helicobacter pylori & WP_042636128.1 \\
\hline & Haemophilus influenza & WP_005663828.1 \\
\hline & Bordetella bronchiseptica & KFJ54879.1 \\
\hline & Neisseria meningitides & WP_002242076.1 \\
\hline \multirow[t]{12}{*}{ DiaA } & Colwellia psychrerythraea & WP_033091889.1 \\
\hline & Yersinia enterocolitica & WP_005174225.1 \\
\hline & Pseudomonas aeruginosa & WP_025299231.1 \\
\hline & Vibrio cholera & WP_032476931.1 \\
\hline & Escherichia coli & WP_004018383.1 \\
\hline & Yersinia pestis & WP_002210146.1 \\
\hline & Franconibacter pulveris & WP_024559311.1 \\
\hline & Salmonella enterica & WP_000893482.1 \\
\hline & Pluralibacter gergoviae & WP_043080845.1 \\
\hline & Erwinia toletana & WP_017801381.1 \\
\hline & Ewingella americana & WP_034786756.1 \\
\hline & Pseudomonas syringae & WP_032629664.1 \\
\hline
\end{tabular}

Ni-NTA affinity resin (Clontech, USA) pre-equilibrated with lysis buffer was used to purify of the target protein. After washing the column and matrix with a ten-column volume of washing buffer ( $50 \mathrm{mM}$ sodium phosphate [pH 8.0 ], $300 \mathrm{mM} \mathrm{NaCl}$, and $35 \mathrm{mM}$ imidazole), the His-tagged protein was eluted using a solution of the same composition but with $300 \mathrm{mM}$ imidazole. Once the His tag was cleaved at $4^{\circ} \mathrm{C}$ overnight with thrombin (Sigma-Aldrich, USA), the resulting protein has an additional GSHM sequence at the N-terminus, an artifact of the cloning. The cleaved protein was loaded onto a Superdex-200 column (GE Healthcare, USA) equilibrated with $20 \mathrm{mM}$ Tris- $\mathrm{HCl}(\mathrm{pH}$ 8.0), $150 \mathrm{mM} \mathrm{NaCl}$, and $1 \mathrm{mM}$ dithiothreitol (DTT). The fractions containing CpsGmhA were collected and concentrated to $25.0 \mathrm{mg} / \mathrm{ml}$ for crystallization trials.

\section{Crystallization, data collection, and structural \\ determination}

The purified CpsGmhA was subjected to an initial crystallization screen using the commercially available crystallization solution kits MCSG I-IV (Microlytic, USA), Wizard Classic I-IV (Emerald Bio, USA), and Classics and Classics II Suite (Qiagen, Germany), following the vapor diffusion method by mixing the protein $(0.8 \mu \mathrm{l})$ and reservoir $(0.8 \mu \mathrm{l})$ solutions. Small crystal granules were obtained within two or three days in $0.1 \mathrm{M}$ Tris- $\mathrm{HCl}(\mathrm{pH}$ 8.5), $0.2 \mathrm{M}$ magnesium chloride, and $30 \%(\mathrm{v} / \mathrm{v})$ PEG400 or $25 \%$ (w/v) PEG3350. The optimum crystallization condition was further refined. Flat hexagonal crystals suitable for X-ray data collection were formed in a hanging drop composed of $1 \mu \mathrm{l}$ of CpsGmhA (25.0 mg/ml), $20 \mathrm{mM}$ Tris- $\mathrm{HCl}, 150 \mathrm{mM} \mathrm{NaCl}$, and 1 $\mathrm{mM}$ DTT, and $1 \mu \mathrm{l}$ of reservoir solution in $0.1 \mathrm{M}$ Tris- $\mathrm{HCl}(\mathrm{pH}$ 7.2), $0.2 \mathrm{M}$ magnesium chloride, and 19\% (v/v) PEG400 against $500 \mu \mathrm{l}$ reservoir. N-paratone was used as a cryoprotec- tant based on the results of a screening experiment. A native data set was collected at $0.97951 \AA$ at the $5 \mathrm{C}$ beamline of the Pohang Light Source (PLS; Korea).

The data were recorded, integrated, and scaled using iMosflm software (Battye et al., 2011). In order to obtain the phase, molecular replacement (MR) was performed using the program MOLREP (Vagin and Teplyakov, 2009), using the homologous structure from $E$. coli DiaA (PDB databank accession number 2YVA; (Keyamura et al., 2007) as the model. A Matthews coefficient of 2.22 and $44.59 \%$ solvent indicated that one molecule was present in an asymmetric unit. After refinement up to $2.8 \AA$ with REFMAC5 (Murshudov et al., 2011), the quality of the final structure was validated using the MOLPROBITY server (Chen et al., 2009) and SFCHECK from the CCP4 package (Vaguine et al., 1999), resulting in a final model with an $R$-factor of $21.0 \%\left(R_{\text {free }} 28.0 \%\right)$. The final refinement statistics are presented in Table 1. Figures and the surface and angle data were generated and calculated using PyMOL (DeLano, 2002).

\section{Sequence analysis}

The amino acid sequences of GmhA and DiaA from C. psychrerythraea strain $34 \mathrm{H}$ and other relevant species were obtained from the National Center for Biotechnology Information (NCBI) database (Table 2). Amino acid sequences were aligned using Clustal X (Chenna et al., 2003). Only well-aligned and conserved alignment sites were extracted from each alignment subset using Gblock (Castresana, 2000). Maximum likelihood $(\mathrm{ML})$ analysis was carried out using RAxML Ver. 7.2.8 (Stamatakis, 2006). RAxML was run under the options GTRCAT and protein GAMMA, a complete random starting tree of 1000 bootstrap replicates (Pattengale et al., 2010). 
$\boldsymbol{A}$

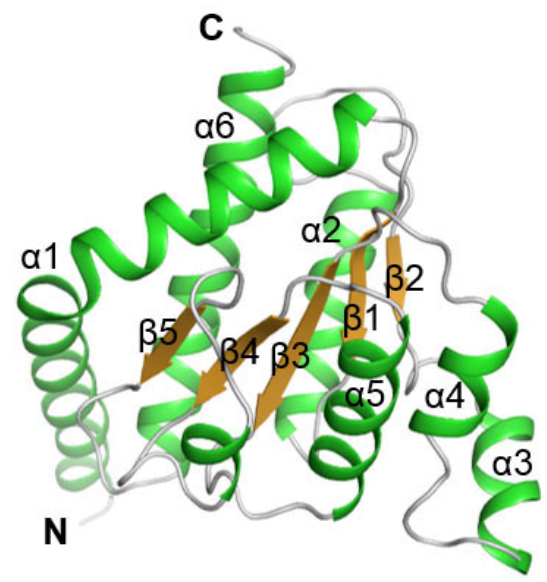

$C$

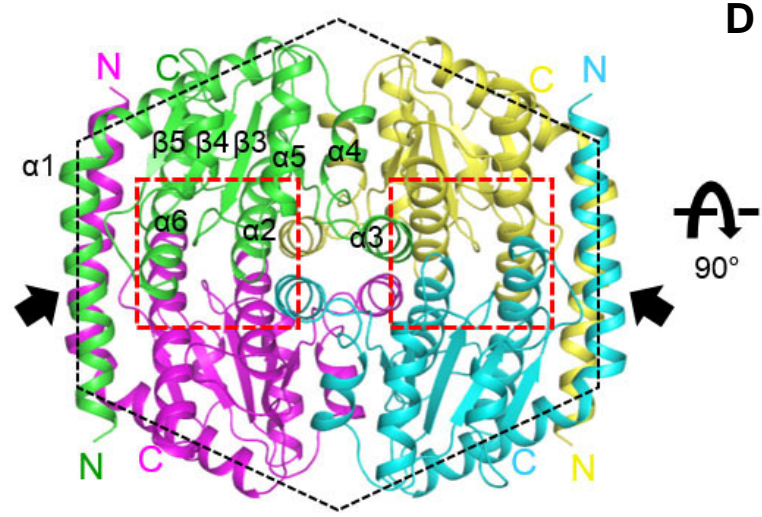

B

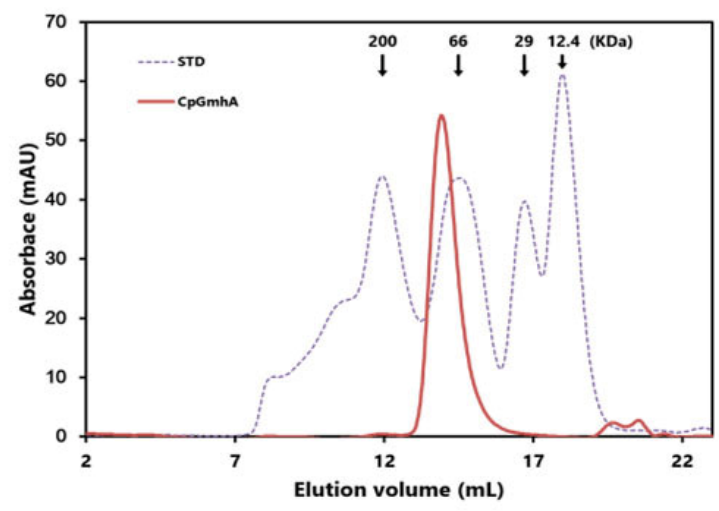

D

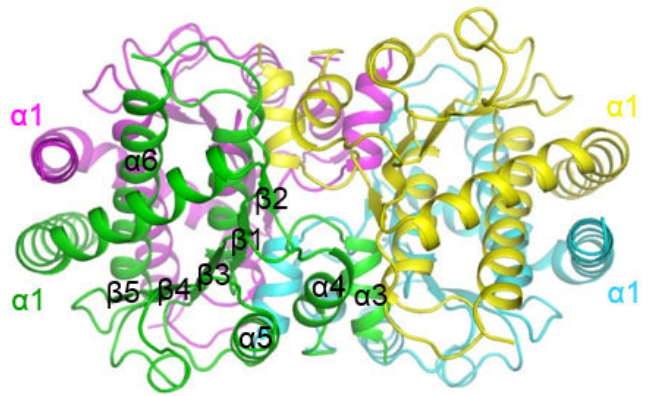

Fig. 1. Overall structure of CpsGmhA. (A) The overall structure of the monomeric CpsGmhA is shown as a cartoon representation. The $\alpha$ helices, $\beta$-sheets, and loops are colored in green, brown, and grey, respectively. (B) Size exclusion chromatogram of CpsGmhA for the measurement of molecular weight. The estimated molecular weight is $84 \mathrm{kDa}$ based on the standard (STD), whereas the calculated molecular weight of the monomer is $20.9 \mathrm{kDa}$. (C) The overall structure of tetrameric CpsGmhA viewed from the top. The four monomers are colored green, yellow, cyan, and magenta, respectively. The active sites are marked with red dotted lines. The black arrows indicate the clamp helical regions. The black dashed hexagon indicates the overall shape by interactions of the $\alpha 1$ helices. (D) Side view of the CpsGmhA tetramer by $90^{\circ}$ rotation over an $\mathrm{X}$-axis from Fig. $1 \mathrm{C}$.

\section{Data deposition}

The atomic coordinates and experimental structure have been deposited in the Protein Data Bank (accession codes 5BY2).

\section{RESULTS}

\section{Overall structure}

The GmhA from C. psychrerythraea strain 34H (CpsGmhA), including the additional serine-histidine residues located before the $\mathrm{N}$-terminus as an artifact of cloning, was crystallized and the structure was determined by X-ray crystallography at a resolution of $2.8 \AA$. The monomeric structure is composed of five central parallel $\beta$-sheets surrounded by six $\alpha$-helices (Fig. 1A). Most residues fit well in the electron density except for residues Tyr93, GIn94, Gln193, Gly194, Asp195, and Ser196. The final model was refined to $R$ and $R_{\text {free }}$ values of $21.0 \%$ and $28.0 \%$, respectively (Table 1). The overall topology of CpsGmhA is very similar to that of the homologous structures from various species, with an average root mean square devia- tion (r.m.s.d.) of $1.3 \AA$ in monomeric $\mathrm{C} \alpha$ (Harmer, 2010; Kim and Shin, 2009; Seetharaman et al., 2006; Taylor et al., 2008). Since one molecule of CpsGmhA was observed in the asymmetric unit, we performed a size exclusion chromatographic analysis. The result indicates that CpsGmhA also exists in tetrameric form in solution, which is in agreement with the results of previous studies (Harmer, 2010; Taylor et al., 2008) (Fig. 1B).

The bulk of helices ( $\alpha 1-\alpha 4$ and $\alpha 6)$ form a tetramer with $a$ broad surface range. Half of the long $\mathrm{N}$-terminal $\alpha 1$ helix interacts with the same region of the neighboring monomers via the hydrophobic residues Met1, Leu2, Ile5, Phe9, lle13, lle17, and lle27, resulting in a four-helix frame around the tetramer (Figs. $1 \mathrm{C}$ and 1D). The four $\alpha 1$-helices in the tetramer form a hexagon tilted at an angle of $120^{\circ}$, which could be important for structural stabilization, because the four $\alpha 1$-helices surrounding the tetramer have broad contacting surfaces that serve as clamps on each of the two subunits (Fig. 1C). Extensive interactions with neighboring molecules support the order of the $\alpha 3$ helix, such that the overall shape has a closed conformation in 
Crystal Structure of Putative CpsGmhA

Hackwon Do et al.

$\boldsymbol{A}$

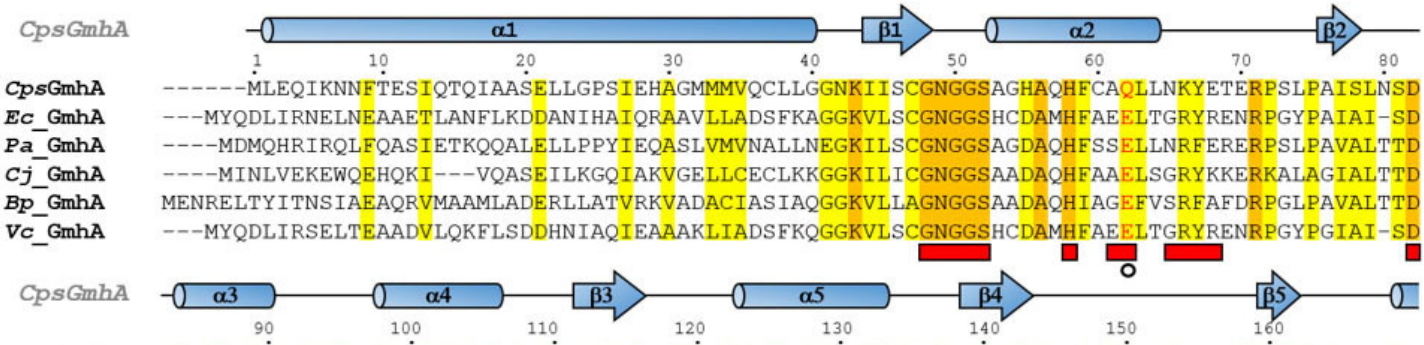

CPsGmhA

EC_GmhA

Pa_GmhA

Cj_GmhA

Bp_GmhA

VC_GmhA

ISTITSIÄNDYOYDEVFS்KOIRALGHNG்DVLLAISTSĠNSRNVVKAIE்SAVSRDIPIİALTGFDGGDISSGLGEGDVEIRVPSAR-TS VSHISCVGNDFGFNDIFSRYVEAVGREGDVLLGISTSGNSANVIKAIAAAREKGMKVITLTGKDGGKMAGTA---DIEIRVPHFGYAD SSTITSIANDYSYNEVFSKQIRALGQPGDVLLAISTSGNSANVIQAIQAAHDREMLVVALTGRDGGGMASLLLPEDVEIRVPSKI-TA TSALSAIGNDYGFEFVFSRQVEALGNEKDVLIGISTSGKSPNVLEALKKAKELNMLCLGLSGKGGGMMNKLC---DHNLVVPSDD-TA TSILTAIGNDYGYEKLFSRQVQALGNEGDVLIGYSTSGKSPNILAAFREAKAKGMTCVGFTGNRGGEMRELC---DLLLEVPSAD-TP PSHLSCVSNDFGYDYVFSRYVEAVGAKGDVLFGLSTSGNSGNILKAIEAAKAKGMKTIALTGKDGGKMAGLA---DVEIRVPHFGYAD

CPsGmhA

CPsGmhA

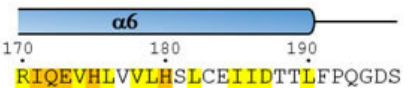

RIOEIHIKVIHILIOLIEKEMVK---

Pa_GmhA RIQEVHLLAIHCLCDLIDRQLFGSEE-

Cj_GmhA RIQEMHILIIHTLCQIIDESF------

BP_GmhA KIQEGHLVLGHIVCGLVEHSIFGKQ--

Vc GmhA

RIQEVHIKIIHIIIQLIEKEMA-----

B

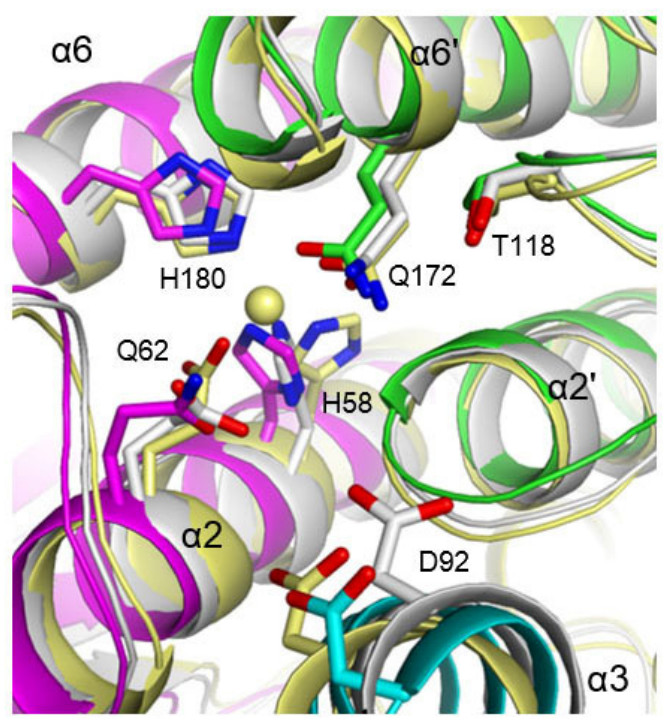

C

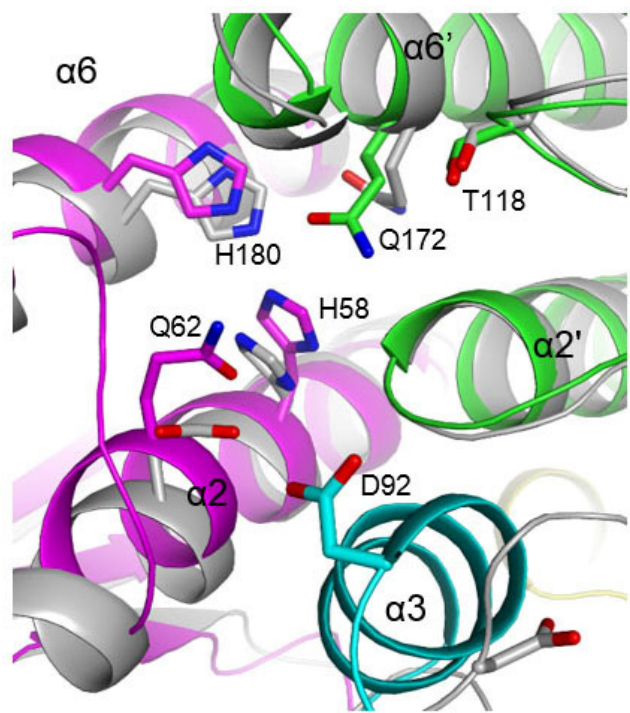

Fig. 2. Active site of CpsGmhA. (A) Structure-based multiple sequence alignment of CpsGmhA with other homologs. Conserved residues are presented in either orange or yellow backgrounds depending on their degree of conservation. The residues composed of the active site are indicated with red rectangles under the sequences. The catalytic residues are marked with open circles. The secondary structure was generated based on the structure of CpsGmhA. Sequences were aligned using Clustal X 2.1 (Chenna et al., 2003) and photographs were generated using the program ESPript (Gouet et al., 1999). (B) Overlay of the catalytic residues of CpsGmhA with those of BpGmhA (PDB id: 2X3Y) and CjGmhA (PDB id: 1TK9). The colors of CpsGmhA are the same as those described for Fig. 1C. BpGmhA and CjGmhA are colored in yellow and grey, respectively. The yellow sphere indicates the zinc ion present in the BpGmhA structure. Oxygen and nitrogen atoms are shown in red and blue, respectively. (C) Overlay of the catalytic residues of CpsGmhA with EcGmhA (PDB id: 2l2W), which is shown in grey.

comparison with homologous structures (see below) (Harmer, 2010; Taylor et al., 2008).

\section{Active site}

The active site of GmhA is mainly composed of four $\alpha$-helices, which are each composed of two equivalent helices $(\alpha 2-\alpha 6$ and $\alpha 2$ '- $\alpha 6^{\prime}$ ) from two subunits. In addition, the conserved residues on the $\alpha 3$ helix and the three loops $(\beta 1-\alpha 2, \alpha 2-\beta 2$, and $\beta 3-\alpha 5)$ support the conformation of the active site (Fig. 2A). The E-Q-H residues, which are located at the $\alpha 2, \alpha 6$ ', and $\alpha 6$ helices, respectively, are strongly involved in catalytic reactions. The E-Q$\mathrm{H}$ residues are highly conserved in the GmhA family; however, 
$\boldsymbol{A}$

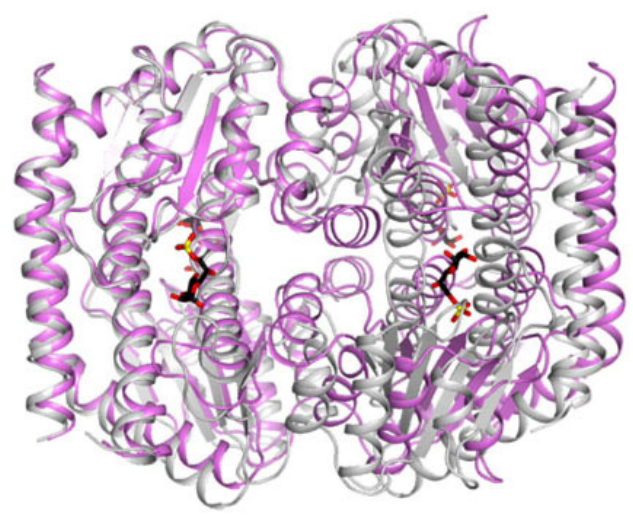

C

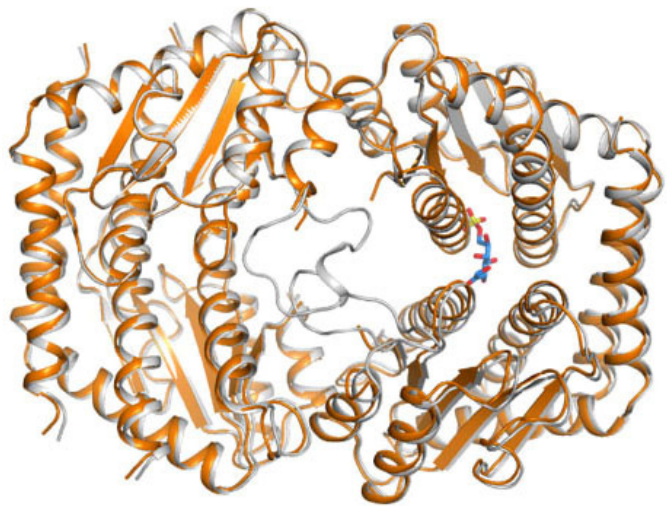

$\boldsymbol{B}$

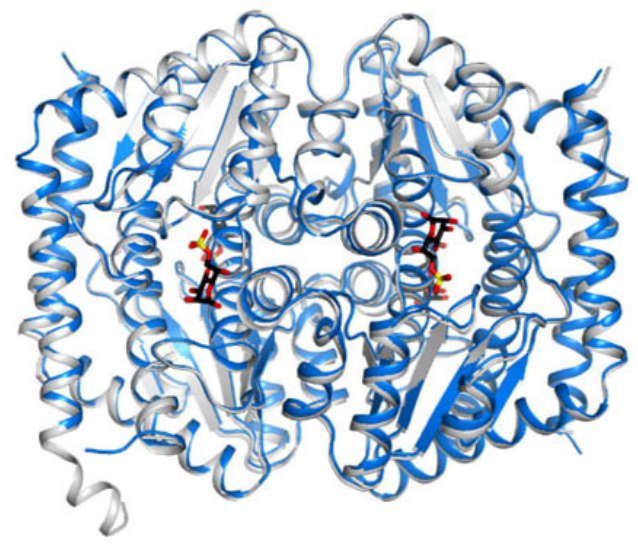

$D$

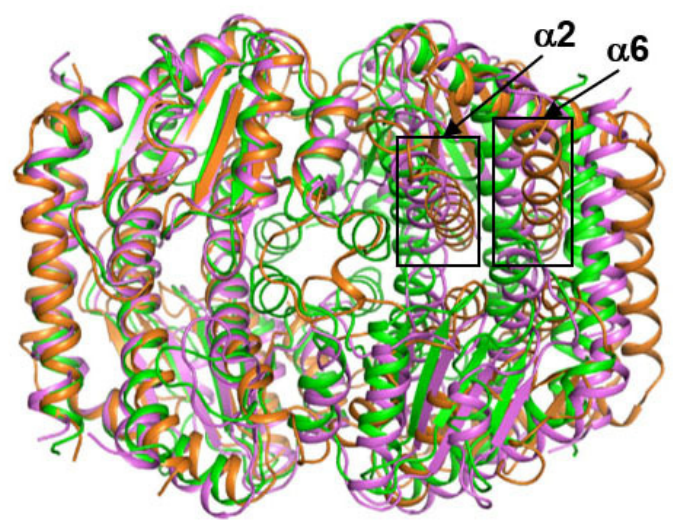

Fig. 3. Comparison of GmhA homologous structures. (A) Overlay of the apo and M7P product-bound GmhA structures from $P$. aeruginosa. The PaGmhA-M7P complex (PDB id: 1X92) and apo PaGmhA (PDB id: 3BJZ) are shown in pink and grey, respectively. The M7P molecules are shown in black with the phosphorous and oxygen atoms in yellow and red, respectively. (B) Overlay of the apo and M7P product-bound GmhA structures from B. pseudomallei. The BpGmhA-M7P complex (PDB id: 2XBL) and apo BpGmhA (PDB id: 2X3Y) are shown in blue and grey, respectively. (C) Overlay of the apo and substrate S7P-bound GmhA structures from E. coli. The EcGmhA-S7P complex (PDB id: 2122) and apo $E c G m h A$ (PDB id: $2 \mathrm{l} 2 \mathrm{~W}$ ) are shown in orange and grey, respectively. The S7P molecules are shown in blue. (D) Overlay of the apo structures of CpsGmhA (green), PaGmhA (pink) and $E_{c} G m h A$ (orange). The tilted $\alpha 2$ and $\alpha 6$ helices of $E c G m h A$ are presented in the black squares.

the glutamate residue is replaced by glutamine at the equivalent position in CpsGmhA (Fig. 2A). Previous mutagenesis studies have shown no GmhA activity in EQ mutants (Harmer, 2010; Taylor et al., 2008), suggesting that CpsGmhA might be more closely related to DiaA this inference is supported by the bioinformatic analysis (see below).

The tetrameric structures of $\mathrm{GmhA}$ can be classified into two distinct conformations, open or closed, depending on whether an additional $\alpha 3^{\prime}$ helix is present in the central region. The closed conformation may allow for the ingress and egress of a substrate and product in solution due to the extension of the solvent accessible area of the active site (Harmer, 2010). Since the conformation of CpsGmhA is closed, we compared the positions of the catalytic residues to those in other $\mathrm{GmhAs}$ with closed conformation from Burkholderia pseudomallei and Campylobacter jejuni (Fig. 2B). Most residues are oriented in a similar manner, except for the Glu63 residue of $C$. jejuni $\mathrm{GmhA}$ (CjGmhA), which is equivalent to the Gln62 residue in CpsGmhA. However, in comparison with those of the E. coli
GmhA (EcGmhA), conformations of the catalytic residues are not well overlaid (Fig. 2C). In particular, the conformation of the Asp94 residue, which is equivalent to Asp92 in CpsGmhA, is quite different in that it faces the opposite side to Asp92. In addition, the positions of the Glu65 and $\mathrm{Gln} 172$ residues (equivalent to Gln62 and Gln172 of CpsGmhA, respectively) are different. Therefore, the conformations of the catalytic residues are affected by whether or not the overall structure is an open or closed conformation.

\section{Comparison with homologous GmhA structures}

Conformational differences between the open and closed may be because of the binding of the product to the active site. For example, the open-conformation structure of $\mathrm{PaGmhA}$ is converted to a closed structure by M7P binding (Taylor et al., 2008) (Fig. 3A). M7P binding drives the $\alpha 4$ helix to move away from the central cavity, resulting in a disordered $\alpha 3$ helix as a result of its interaction with the $\alpha 2$ of the other subunit. However, the $B$. pseudomallei and E. coli GmhA structures are not changed 
A

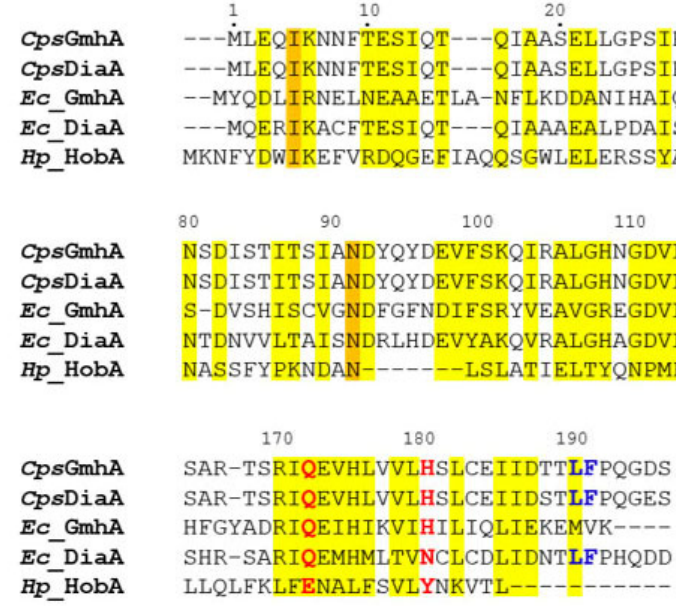

B

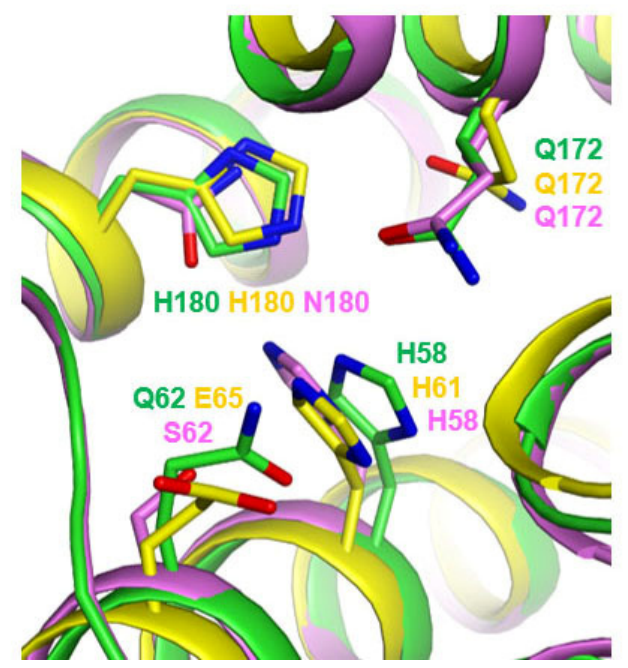

$30 \quad 40$

50

60

70

GंGSAGHAQHF $\dot{C} A Q L L N K Y E T \dot{R} P S L P A I S L$ AGMMMVQCLLGGNKI ISCGNGGSAGHAQHFCAQLLNKYETERPSLPAISL

STSGNSRNVVKAIESAVSRDIPIIALTGFDGGDISGLLGEGDVEIRVP IESAVSRDIPIIALTGFDGGDISGLLGEGDVEIRVP 作 STRGNSRDIVKAVEAAVTRDMT IVALTGYDGGELAGLLGPQDVEIRIP VGKI EN-----EGLKTLLLSKI PSFLWLFEELKEDCLLLKEHDSLLDYK 


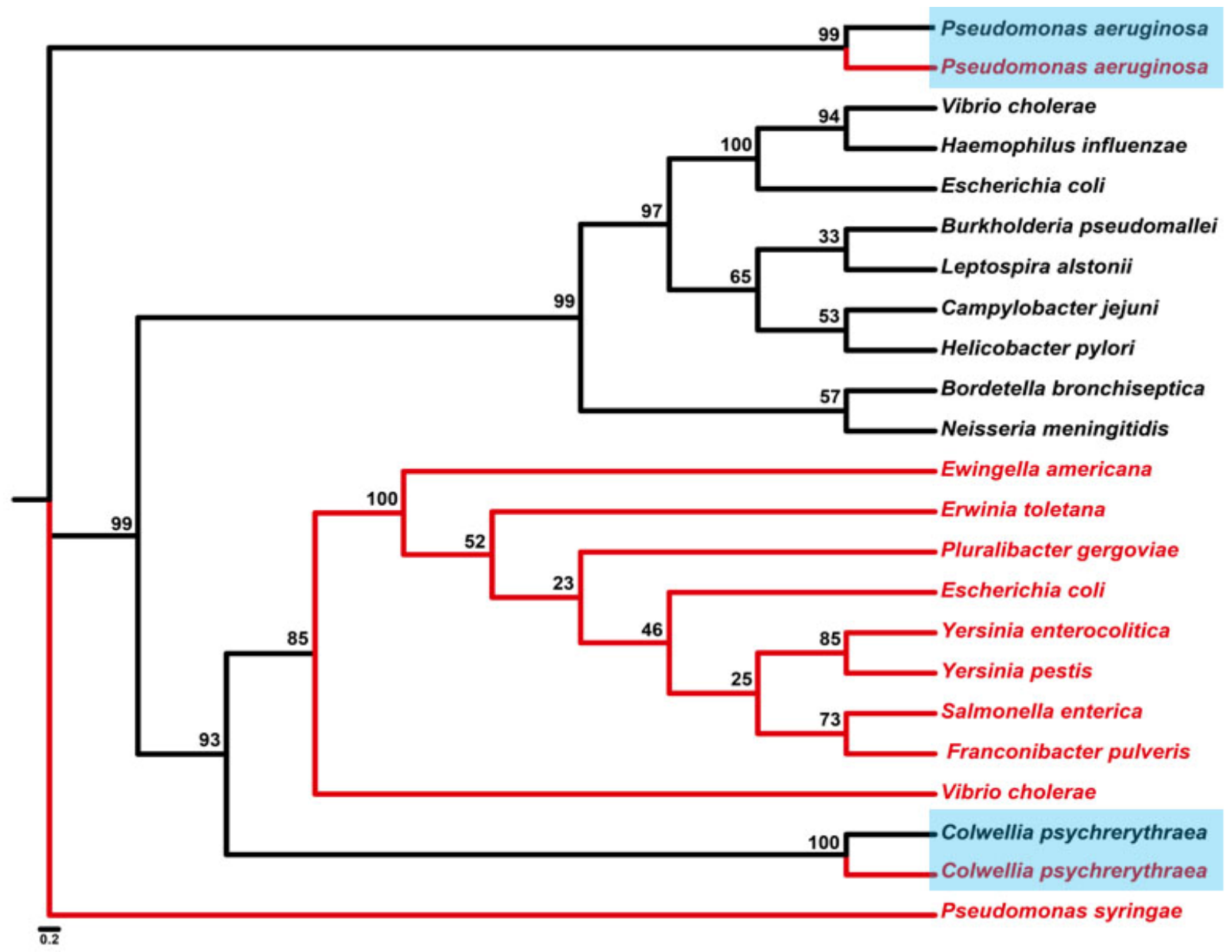

Fig. 5. Phylogenetic analysis of GmhA and DiaA. Phylogenetic relationships of DiaA and GmhA based on their amino acids. The consensus cladogram inferred from the amino acid sequence data of DiaA and GmhA using maximum likelihood (ML) analysis under GTRCAT and protein GAMMA models. The group of DiaA is highlighted in red color. The paired GmhA and DiaA from C. psychrerythraea and $P$. aeruginosa are highlighted in sky blue. The numbers above the branches indicate bootstrap percentages.

Next, we compared the structural similarity among the four structures. Structural comparisons show that CpsGmhA has higher similarity to $E c D i a A$ with a r.m.s.d of $0.7 \AA$, but relatively low similarity to both EcGmhA and $H p H o b A$ with an r.m.s.d of $1.7 \AA$ and $3.7 \AA$, respectively. Thus, the overall tetrameric structures of CpsGmhA and EcDiaA are completely overlaid (Fig. $4 C)$. To investigate the sequence-based relationship between CpsGmhA and CpsDiaA, we performed a phylogenetic analysis of GmhA and DiaA (Fig. 5). GmhA and DiaA could be divided into two distinct groups. However, we found that GmhA and DiaA from C. psychrerythraea and Pseudomonas aeruginosa are not clearly separated. Indeed, GmhA and DiaA from the two species showed over $98 \%$ similarity because they differ in only two or three residues. This result indicates that $P a D i a A$ could be classified as an isotype of $P a G m h A$ and $C p s G m h A$ as an isotype of CpsDiaA. Taken together, the structural and sequence similarities of CpsGmhA, CpsDiaA and EcDiaA may provide insight into the functional role of $\mathrm{GmhA}$ as an isotype of DiaA in C. psychrerythraea.

\section{DISCUSSION}

To date, eight crystal structures of GmhA have been reported from various mesophilic species (Harmer, 2010; Kim and Shin, 2009; Seetharaman et al., 2006; Taylor et al., 2008). The overall structures are divided into two forms: open and closed. The conformations of the apo-structures are also variable in that the GmhAs of E. coli, Vibrio cholera, and $P$. aeruginosa are open, whereas those of $B$. pseudomallei, $C$. jejuni, and C. psychrerythraea are closed. The overall structure of psychrophilic CpsGmhA is very similar to the homologous structures of the closed forms. Previous studies of GmhA crystal structures demonstrate that either two or four molecules are contained in the asymmetric unit. However, only one molecule of CpsGmhA was found in the asymmetric unit. The biologically reliable tetrameric structure was revealed by the $C 222$ symmetry operation. Additional analysis of oligomerization using the program PDBePISA (v1.47) further indicated that biological assembly of the protein results in a tetramer (Krissinel and Henrick, 2007).

The reaction mechanisms of GmhA have been proposed by complex structures with either product or substrate (Harmer, 2010; Taylor et al., 2008). To date, there have been three GmhA complex structures reported in either substrate form or productbound form. The substrate S7P is only present in the EcGmhA 
structure as an open conformation, and the product M7P is found bound in closed structures from $P$. aeruginosa and $B$. pseudomallei (Harmer, 2010; Taylor et al., 2008). Moreover, only one subunit of the tetrameric EcGmhA contains the substrate S7P, whereas BpGmhA and PaGmhA both have M7P products in each of the four subunits (Fig. 4C). Thus, the two subunits are not symmetrical about the vertical axis, as is the case with other GmhA structures. This may indicate that a local conformational change occurs upon substrate binding. Howev$\mathrm{er}$, there was no difference in symmetry between the protein and its apo-structure. On the other hand, it is also likely that a change in only one molecule of the substrate would not induce an overall conformational change.

As shown in the previous section, only CpsGmhA contains a catalytic glutamine, rather than a glutamate residue at the equivalent position (GIn62) in the active site. Therefore, CpsGmhA may not have isomerase activity, based on mutagenesis data reported previously (Harmer, 2010; Taylor et al., 2008). Furthermore, based on sequence identity, CpsGmhA is more closely related to EcDiaA $(62.2 \%$ identical) than to EcGmhA (37\% identical) or HpHobA (13.9\% identical). Interestingly, CpsGmhA has the two signature residues, Leu190 and Phe191, in common with those of EcDiaA, which are critical for DnaA binding, whereas EcGmhA does not contain these residues (Fig. 5A) (Keyamura et al., 2007). In addition, there are only three residue discrepancies between CpsGmhA and CpsDiaA, and therefore no significant functional differences would be expected. Since a functional study of CpsGmhA has not yet been done, we propose that the CpsGmhA protein could be a putative isotype of CpsDiaA. Taken together, further investigation of $C p s G m h A$ is required to confirm the functional similarity with CpsDiaA.

\section{ACKNOWLEDGMENTS}

We would like to thank the staff at the X-ray core facility of the Korea Basic Science Institute (KBSI; Ochang, Korea) and Yeon-Gil Kim, the beamline staff at PLS-5C of the Pohang Light Source (Pohang, Korea) for the data collection. This work was supported by the Korea Polar Research Institute (KOPRI; grant No. PE14330 to JHL) and Basic Science Research Program through the National Research Foundation of Korea (NRF) funded by the Ministry of Education, Science and Technology to JHC (2013R1A1A1061391).

\section{REFERENCES}

Battye, T.G., Kontogiannis, L., Johnson, O., Powell, H.R., and Leslie, A.G. (2011). iMOSFLM: a new graphical interface for diffractionimage processing with MOSFLM. Acta Crystallogr. Sect D: Biol. Crystallogr. 67, 271-281.

Bazaka, K., Crawford, R.J., Nazarenko, E.L., and Ivanova, E.P. (2011). Bacterial extracellular polysaccharides. In Bacterial adhesion (Springer), pp. 213-226.

Brooke, J.S., and Valvano, M.A. (1996). Biosynthesis of inner core lipopolysaccharide in enteric bacteria identification and characterization of a conserved phosphoheptose isomerase. J. Biol. Chem. 271, 3608-3614.

Carillo, S., Casillo, A., Pieretti, G., Parrilli, E., Sannino, F., BayerGiraldi, M., Cosconati, S., Novellino, E., Ewert, M., Deming, J.W. et al. (2015). A unique capsular polysaccharide structure from the psychrophilic marine bacterium Colwellia psychrerythraea $34 \mathrm{H}$ that mimics antifreeze (glyco)proteins. J. Am. Chem. Soc. 137, 179-189.

Castresana, J. (2000). Selection of conserved blocks from multiple alignments for their use in phylogenetic analysis. Mol. Biol. Evol. 17, 540-552.

Chattopadhyay, M. (2006). Mechanism of bacterial adaptation to low temperature. J. Biosci. 31, 157-165.
Chen, V.B., Arendall, W.B., Headd, J.J., Keedy, D.A., Immormino, R.M., Kapral, G.J., Murray, L.W., Richardson, J.S., and Richardson, D.C. (2009). MolProbity: all-atom structure validation for macromolecular crystallography. Acta Crystallogr. Sect D: Biol Crystallogr. 66, 12-21.

Chenna, R., Sugawara, H., Koike, T., Lopez, R., Gibson, T.J., Higgins, D.G., and Thompson, J.D. (2003). Multiple sequence alignment with the Clustal series of programs. Nucleic Acids Res. $31,3497-3500$.

DeLano, W.L. (2002). The PyMOL molecular graphics system.

Feller, G. (2013). Psychrophilic enzymes: from folding to function and biotechnology. Scientifica (Cairo) 2013, 512840.

Feller, G., and Gerday, C. (2003). Psychrophilic enzymes: hot topics in cold adaptation. Nat. Rev. Microbiol. 1, 200-208.

Gouet, P., Courcelle, E., and Stuart, D.I. (1999). ESPript: analysis of multiple sequence alignments in PostScript. Bioinformatics 15 , 305-308.

Harmer, N.J. (2010). The structure of sedoheptulose-7-phosphate isomerase from Burkholderia pseudomallei reveals a zinc binding site at the heart of the active site. J. Mol. Biol. 400, 379-392.

Holm, L., and Rosenstrom, P. (2010). Dali server: conservation mapping in 3D. Nucleic Acids Res. 38, W545-549.

Huston, A.L., Krieger-Brockett, B.B., and Deming, J.W. (2000). Remarkably low temperature optima for extracellular enzyme activity from Arctic bacteria and sea ice. Environ. Microbiol. 2, 383-388.

Huston, A.L., Methe, B., and Deming, J.W. (2004). Purification, characterization, and sequencing of an extracellular cold-active aminopeptidase produced by marine psychrophile Colwellia psychrerythraea strain $34 \mathrm{H}$. Appl. Environ. Microbiol. 70, 33213328.

Ishida, T., Akimitsu, N., Kashioka, T., Hatano, M., Kubota, T., Ogata, Y., Sekimizu, K., and Katayama, T. (2004). DiaA, a novel DnaAbinding protein, ensures the timely initiation of Escherichia coli chromosome replication. J. Biol. Chem. 279, 45546-45555.

Junge, K., Eicken, H., Swanson, B.D., and Deming, J.W. (2006). Bacterial incorporation of leucine into protein down to -20 degrees $\mathrm{C}$ with evidence for potential activity in sub-eutectic saline ice formations. Cryobiology 52, 417-429.

Keyamura, K., Fujikawa, N., Ishida, T., Ozaki, S., Su'etsugu, M., Fujimitsu, K., Kagawa, W., Yokoyama, S., Kurumizaka, H., and Katayama, T. (2007). The interaction of DiaA and DnaA regulates the replication cycle in $E$. coli by directly promoting ATP-DnaAspecific initiation complexes. Genes Dev. 21, 2083-2099.

Kim, M.S., and Shin, D.H. (2009). A preliminary X-ray study of sedoheptulose-7-phosphate isomerase from Burkholderia pseudomallei. Acta Crystallogr. Sect. F Struct. Biol. Cryst. Commun. 65, 1110-1112.

Kneidinger, B., Graninger, M., Puchberger, M., Kosma, P., and Messner, P. (2001). Biosynthesis of nucleotide-activated Dglycero-D-manno-heptose. J. Biol. Chem. 276, 20935-20944.

Krembs, C.e., Eicken, H., Junge, K., and Deming, J. (2002). High concentrations of exopolymeric substances in Arctic winter sea ice: implications for the polar ocean carbon cycle and cryoprotection of diatoms. Deep Sea Res. Part I 49, 2163-2181.

Krissinel, E., and Henrick, K. (2007). Inference of macromolecular assemblies from crystalline state. J. Mol. Biol. 372, 774-797.

Marx, J.G., Carpenter, S.D., and Deming, J.W. (2009). Production of cryoprotectant extracellular polysaccharide substances (EPS) by the marine psychrophilic bacterium Colwellia psychrerythraea strain 34H under extreme conditions. Can J. Microbiol. 55, 63-72.

Methe, B.A., Nelson, K.E., Deming, J.W., Momen, B., Melamud, E., Zhang, X.J., Moult, J., Madupu, R., Nelson, W.C., Dodson, R.J., et al. (2005). The psychrophilic lifestyle as revealed by the genome sequence of Colwellia psychrerythraea $34 \mathrm{H}$ through genomic and proteomic analyses. Proc. Natl. Acad. Sci. USA 102, 10913-10918.

Murshudov, G.N., Skubák, P., Lebedev, A.A., Pannu, N.S., Steiner, R.A., Nicholls, R.A., Winn, M.D., Long, F., and Vagin, A.A. (2011). REFMAC5 for the refinement of macromolecular crystal structures. Acta Crystallogr. Sect D Biol. Crystallogr. 67, 355-367.

Natrajan, G., Hall, D.R., Thompson, A.C., Gutsche, I., and Terradot, L. (2007). Structural similarity between the DnaA-binding proteins HobA (HP1230) from Helicobacter pylori and DiaA from Escherichia coli. Mol. Microbiol. 65, 995-1005.

Nikaido, H. (2003). Molecular basis of bacterial outer membrane 
permeability revisited. Microbiol. Mol. Biol. Rev. 67, 593-656.

Pattengale, N.D., Alipour, M., Bininda-Emonds, O.R., Moret, B.M., and Stamatakis, A. (2010). How many bootstrap replicates are necessary? J. Comput. Biol. 17, 337-354.

Pikuta, E.V., Hoover, R.B., and Tang, J. (2007). Microbial extremophiles at the limits of life. Crit. Rev. Microbiol. 33, 183-209.

Raetz, C.R., and Whitfield, C. (2002). Lipopolysaccharide endotoxins. Annu. Rev. Biochem. 71, 635-700.

Russell, N.J. (1990). Cold adaptation of microorganisms. Philos. Trans. R Soc. Lond. B. Biol. Sci. 326, 595-608, discussion 608511.

Russell, N.J. (1998). Molecular adaptations in psychrophilic bacteria: potential for biotechnological applications. Adv. Biochem. Eng. Biotechnol. 61, 1-21.

Seetharaman, J., Rajashankar, K.R., Solorzano, V., Kniewel, R., Lima, C.D., Bonanno, J.B., Burley, S.K., and Swaminathan, S. (2006). Crystal structures of two putative phosphoheptose isomerases. Proteins 63, 1092-1096.

Shen, B., Hohmann, S., Jensen, R.G., and Bohnert, H.J. (1999). Roles of sugar alcohols in osmotic stress adaptation. Replacement of glycerol by mannitol and sorbitol in yeast. Plant
Physiol. 121, 45-52

Stamatakis, A. (2006). RAxML-VI-HPC: maximum likelihood-based phylogenetic analyses with thousands of taxa and mixed models. Bioinformatics 22, 2688-2690.

Taylor, P.L., Blakely, K.M., de Leon, G.P., Walker, J.R., McArthur, F., Evdokimova, E., Zhang, K., Valvano, M.A., Wright, G.D., and Junop, M.S. (2008). Structure and function of sedoheptulose-7phosphate isomerase, a critical enzyme for lipopolysaccharide biosynthesis and a target for antibiotic adjuvants. J. Biol. Chem. 283, 2835-2845.

Vagin, A., and Teplyakov, A. (2009). Molecular replacement with MOLREP. Acta Crystallogr. Sect. D. Biol. Crystallogr. 66, 22-25.

Vaguine, A.A., Richelle, J., and Wodak, S. (1999). SFCHECK: a unified set of procedures for evaluating the quality of macromolecular structure-factor data and their agreement with the atomic model. Acta Crystallogr. Sect D. Biol. Crystallogr. 55, 191-205.

Wells, L.E., and Deming, J.W. (2006). Characterization of a coldactive bacteriophage on two psychrophilic marine hosts. Aquat. Microb. Ecol. 45, 15-29. 